\section{Síndrome Burnout en cirujanos dentistas de hospitales de Lima Metropolitana}

Burnout syndrome in dental surgeons from Lima Metropolitan hospitals

\begin{abstract}
Resumen
La investigación fue desarrollada en una muestra aleatoria probabilística de 117 cirujanos dentistas que laboran en hospitales de las Fuerzas Armadas, Seguridad Social y MINSA (Ministerio de Salud) de Lima y Callao, durante los meses de marzo y abril de 2008, con el objetivo de determinar los niveles del Síndrome Burnout en esta población y su asociación con algunas variables sociodemográficas, laborales y de salud general. Para el estudio, se aplicó el cuestionario Maslash Burnout Inventory y una ficha de datos personales. Los resultados muestran que el 44,6 \% de los cirujanos dentistas presenta Síndrome Burnout en nivel severo y se encontró asociaciones estadísticamente significativas con las variables sexo y presencia de problemas de salud.
\end{abstract}

Palabras clave: Síndrome Burnout, estrés laboral, despersonalización, desgaste psíquico, agotamiento emocional.

\begin{abstract}
This research was carried out in a probabilistic randomized sample of 117 dental surgeons who work in hospitals from the Armed Forces, Social Security and the Ministry of Health (MINSA) from Lima and El Callao, during the months of March and April 2008. Its objective was to determine the levels of the Burnout Syndrome in this population and its association with some socio-demographic, labor and general health variables. The Maslash Burnout Inventory questionnaire, as well as a personal data sheet were applied for such study. The results show that $44.6 \%$ of the dental surgeons present severe-level Burnout Syndrome and statistically significant associations were found with sex and the presence of health problems as variables.
\end{abstract}

Key words: Burnout Syndrome, occupational stress, depersonalization, psychic exhaustion, emotional exhaustion.

\section{Ronald Robles Velásquez ${ }^{1}$ Lita Cáceres Gutiérrez ${ }^{2}$}

\author{
Egresado de la UNMSM. C.D. Práctica priva- \\ da. \\ 2 Profesora Asociada del Departamento de Esto- \\ matología Preventiva y Social de la Facultad de \\ Odontología, UNMSM \\ *Tesis para optar el Título de Cirujano Dentista \\ Correspondencia: \\ C.D. Ronald Alberto Robles Velásquez \\ Jr. Diez Canseco 3446, Urb. Condevilla. San \\ Martín de Porres, Lima 13. Perú. \\ (Teléfono: 997279 036) \\ Correo electrónico: ortodonciabasica@gmail.com
}

\section{Introducción}

El Síndrome Burnout es una respuesta al estrés laboral crónico de gran prevalencia en los profesionales de la salud, integrada por sentimientos y actitudes negativos hacia las personas con las que se trabaja (actitudes de despersonalización) y hacia el propio rol profesional (falta de realización personal en el trabajo) así como por agotamiento emocional $^{1}$. La despersonalización se refiere al desarrollo de actitudes, sentimientos y respuestas negativas, distantes y frías hacia los pacientes; la falta de realización personal en el trabajo se refiere a la tendencia de los profesionales de la salud a evaluarse negativamente afectando la habilidad para realizar el trabajo y el trato con los pacientes, así mismo se sienten descontentos consigo mis- mos e insatisfechos con sus resultados laborales; el agotamiento emocional es una disminución de la energía o los recursos emocionales propios, debido al contacto diario y mantenido con los pacientes. $^{2}$

Las investigaciones dan al Síndrome Burnout diversas denominaciones, tales como: Síndrome de quemarse por el trabajo, Síndrome del quemado, desgaste psíquico en el trabajo, desgaste profesional, desgaste ocupacional, estrés laboral asistencial o estrés profesional; sin embargo todas ellas describen el mismo fenómeno. ${ }^{3}$ La mayoría de los estudios realizados recogen datos epidemiológicos del Síndrome Burnout en médicos, enfermeras y docentes; sin embargo, existe un vacío en el conocimiento científico respecto al Síndrome Burnout en los cirujanos dentistas, quienes también están sometidos a condiciones laborales que originan un elevado estrés.

Por ello, con el objetivo de contribuir a la elaboración de un perfil del riesgo de padecer el Síndrome Burnout en profesionales cirujanos dentistas, se realizó este estudio que busca determinar los niveles Síndrome Burnout de los cirujanos dentistas que trabajan en los hospitales de las Fuerzas Armadas y Policiales, de la Seguridad Social y del Ministerio de Salud (MINSA).

\section{Material y Método}

El estudio es descriptivo y transversal. La población estuvo constituida por todos los Cirujanos Dentistas que laboran en los hospitales de Lima y Callao que pertenecen a las Fuerzas Armadas y Policiales, al MINSA y a la Seguridad 
Social $(\mathrm{N}=347)$. El tamaño de la muestra ascendió a 117 casos y fue calculado de acuerdo con la fórmula de muestras para población límite y variable cualitativa. Se establecieron como parámetros un nivel de ocurrencia de $50 \%$, un margen de error de $5 \%$ y un nivel de confianza de $95 \%$.

Dada la naturaleza del estudio, se desarrolló un muestreo probabilístico por conglomerados, bi-etapico, de acuerdo con el siguiente procedimiento:

En la primera etapa, se constituyó un marco muestral con el listado de hospitales de las Fuerzas Armadas y Policiales, del MINSA y de la Seguridad Social en Lima y Callao. En este momento, se utilizó un muestreo por conglomerados, siendo agrupados los hospitales en estratos de acuerdo a la cantidad de Cirujanos Dentistas que en ellos laboran, a partir de los cuales se eligieron de manera aleatoria los hospitales que participaron en el estudio. En la segunda etapa, en cada hospital seleccionado se eligieron de forma aleatoria los profesionales considerados en el estudio.

Están incluidos en el estudio los cirujanos dentistas que trabajan en hospitales de las Fuerzas Armadas, Policiales, Seguridad Social y MINSA en Lima y Callao; además, los cirujanos dentistas que se encuentran en actividad durante la etapa de recolección de datos y que accedan voluntariamente a participar en el estudio.

Están excluidos los cirujanos dentistas que no están en contacto directo y constante con los pacientes.

Los instrumentos de recolección de datos son:

Una ficha de datos personales, que recoge información de las características generales y específicas del cirujano dentista según las variables del estudio.

El Cuestionario "Maslach Burnout Inventory", que evaluó las tres dimensiones fundamentales del Síndrome Burnout: agotamiento emocional, despersonalización y realización personal. Para evaluar los puntajes del cuestionario, se utilizó el Modelo de Fases de Golembiewski, en el cual se toma como referencia la puntuación en las tres escalas del Maslach Burnout Inventory y tras dicotomizarlas en función de la media, los sujetos son asignados a un grupo en cada escala (alto vs. bajo) según la puntuación obtenida. La combinación de la puntuación obtenida en las tres escalas determina la fase a la que es asignado el sujeto. Se consideraría Burnout leve en las fases I, II, III; Burnout moderado en las fases IV o V; mientras que esta-

Tabla 1. Modelo de las fases de Golembiewski

\begin{tabular}{|c|c|c|c|c|c|c|c|c|}
\hline \multirow{2}{*}{ Variables } & \multicolumn{8}{|c|}{ Fases o Estados del Síndrome de Burnout } \\
\hline & $\mathbf{I}$ & II & III & IV & v & VI & VII & VIII \\
\hline Despersonalización & Bajo & Alto & Bajo & Alto & Bajo & Alto & Bajo & Alto \\
\hline $\begin{array}{l}\text { Falta de realización } \\
\text { personal en el trabajo }\end{array}$ & Bajo & Bajo & Alto & Alto & Bajo & Bajo & Alto & Alto \\
\hline Agotamiento & Bajo & Bajo & Bajo & Bajo & Alto & Alto & Alto & Alto \\
\hline
\end{tabular}

ríamos ante Burnout severo en las fases VI, VII y VIII ${ }^{4}$. Tabla 1.

Con esta información, se procedió al análisis estadístico correspondiente: utilizando frecuencias, porcentajes y la prueba Chi cuadrado.

\section{Resultados}

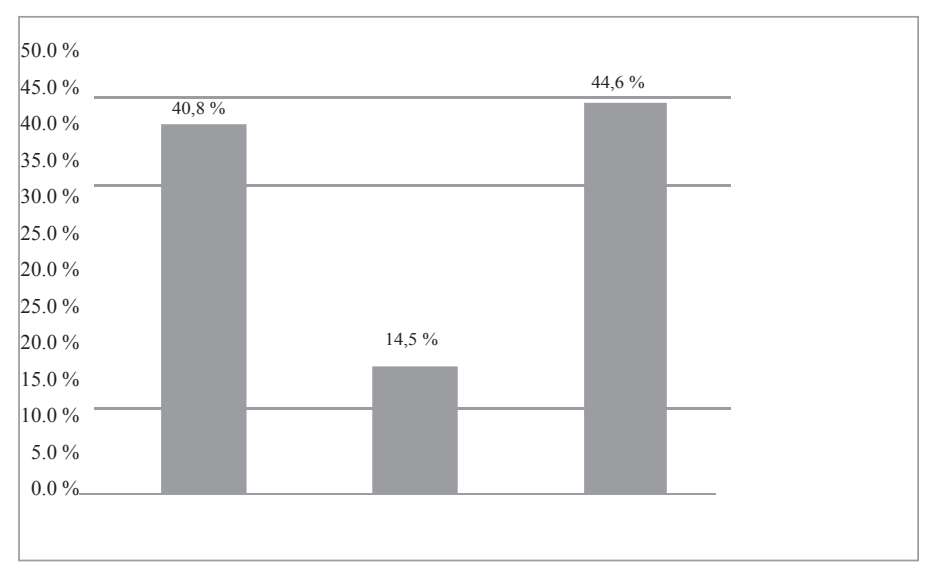

Fig 1. Clasificación de cirujanos dentistas según los niveles del Síndrome Burnout, Lima, 2008.

emocional, despersonalización y falta de realización personal; o dos de ellos incluyendo al agotamiento emocional.

Al analizar los niveles del Síndrome Burnout de acuerdo al tipo de hospital de procedencia, observamos que el nivel severo predomina en los hospitales de las Fuerzas Armadas y Policiales con el $50,1 \%$, y en los hospitales de la Se-

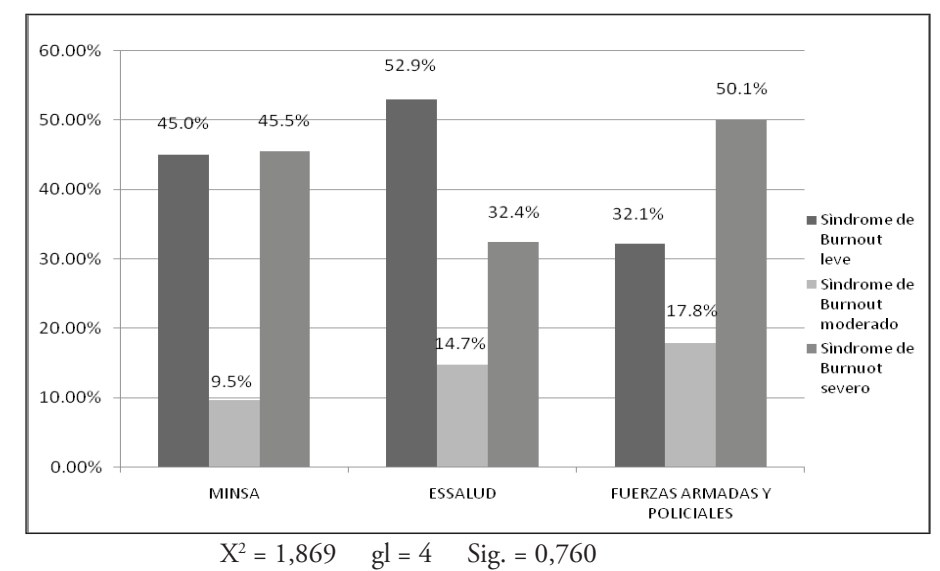

Fig 2. Clasificación de cirujanos dentistas según los niveles del Síndrome Burnout y el tipo de hospital de procedencia, Lima, 2008.
Los resultados muestran que el $44,6 \%$ de los cirujanos dentistas presenta el Síndrome Burnout en un nivel severo, el $14,5 \%$ lo presenta en un nivel moderado y el $40,8 \%$ lo presenta en un nivel leve. Se considera que padecen el Síndrome Burnout solo los que lo tienen en un nivel severo, porque en este nivel se presentan elevados los tres síntomas principales del síndrome: agotamiento

guridad Social predomina el nivel leve con el 52,9\%. Sin embargo, no hubo asociación significativa entre los niveles del Síndrome Burnout y el tipo de hospital de procedencia de los cirujanos dentistas $(\mathrm{P}=0,760)$. Al hacer el análisis de los resultados, se aprecia que entre las mujeres predomina el Síndrome Burnout severo $(65,6 \%)$ y en los varones, el Síndrome Burnout leve (48,3 


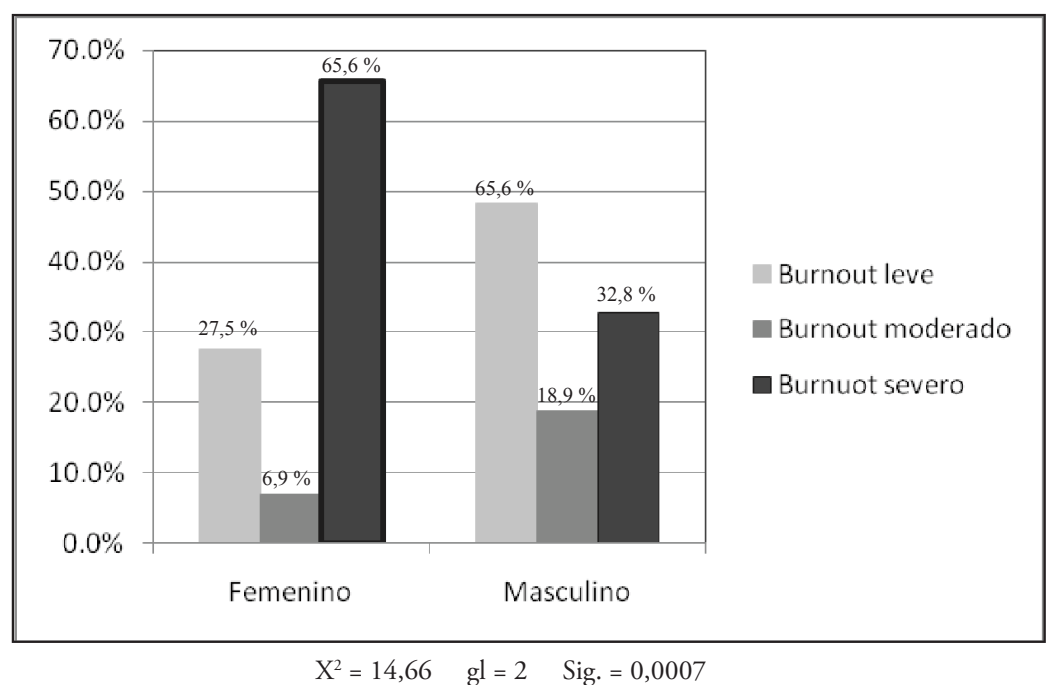

Fig 3. Clasificación de cirujanos dentistas según el sexo y los niveles del Síndrome Burnout, Lima, 2008.
$\%)$, encontrándose una asociación significativa $(\mathrm{P}=0,0007)$ entre el sexo y los niveles del Síndrome Burnout.La presencia de problemas de salud y los niveles del Síndrome Burnout en los cirujanos dentistas presentan una asociación significativa $(\mathrm{P}=0,0169)$. Es así que en los cirujanos dentistas que refieren presentar algún problema de salud (de tipo muscular, de columna, sobrepeso, auditivos, visuales y otros), predomina el Síndrome Burnout alto (49,4\%).

\section{Discusión}

El porcentaje del Síndrome Burnout severo alcanza el 44,6 \% de los cirujanos dentistas en hospitales de las Fuerzas Armadas y Policiales, de la Seguridad Social y del MINSA. Este resultado es similar al obtenido por Cedeño $^{5}$ y Rendulich 6 . Sin embargo, existen estudios realizados en médicos de la ciudad de Tacna ${ }^{7}$ y del Hospital Universitario de Valle ${ }^{8}$, en los cuales más del $52 \%$ tiene criterios de severidad del síndrome. Esto podría relacionarse a las mayores responsabilidades sobre la vida del paciente que recaen sobre los médicos. ${ }^{9}$

Los hospitales de las Fuerzas Armadas y Policiales, de la Seguridad Social y del MINSA presentan características propias en el ámbito laboral y organizacional. Gil-Monte afirma que el entorno laboral y las condiciones de trabajo son los únicos factores que intervienen en la etiología del Síndrome Burnout; ${ }^{10}$ estudios como el de GilMonte y Zurriaga ${ }^{11}$ y Mesquita y col. ${ }^{12}$ sugieren que el Síndrome Burnout estaría relacionado con factores organizacionales. Por esto se analizó los niveles del Síndrome Burnout de acuerdo al tipo de hospital de procedencia, determinándose que no existe una asociación estadísticamente significativa entre los niveles del Síndrome de Burnout y el tipo de hospital de procedencia $(\mathrm{P}=0,760)$; sin embargo, se puede observar que el Síndrome Burnout severo predomina en mayor medida en los hospitales de las Fuerzas Armadas y Policiales (50,1 $\%)$.

Se determinó una asociación estadísticamente significativa entre los niveles de Síndrome Burnout y el sexo de los cirujanos dentistas $(\mathrm{P}=0,0007)$, predominando el Síndrome Burnout severo en el sexo femenino (65,6\%), lo cual concuerda con las afirmaciones realizadas en los estudios de Cedeño ${ }^{13}$ y Gil-Monte. ${ }^{14}$ Sin embargo, algunas investigaciones han encontrado mayo- res niveles del Síndrome Burnout en los varones ${ }^{15}$ y otros no han encontrado asociaciones significativas. ${ }^{7,16,8}$

Las explicaciones a estas diferencias pueden hallarse en los diferentes procesos de socialización seguidos para los roles femenino y masculino, y su interacción con los perfiles y requisitos de las distintas ocupaciones. ${ }^{17}$

Existen referencias de la relación entre la presencia de problemas de salud y el Síndrome Burnout, ${ }^{18,6}$ en nuestro estudio esta asociación significativa se manifiesta nuevamente $(\mathrm{P}=0,0169)$ pues en los cirujanos dentistas que refieren presentar problemas de salud predomina el Síndrome Burnout severo (49,4 $\%)$.

\section{Conclusiones}

El 44,6 \% de los cirujanos dentistas entrevistados presenta el Síndrome Burnout en un nivel severo, el $14,5 \%$ lo presenta en un nivel moderado y 40,8 $\%$ lo presenta en un nivel leve.

En los hospitales de las Fuerzas Armadas y Policiales predomina el Síndrome Burnout en un nivel severo (50,1\%).

Los cirujanos dentistas de sexo femenino presentan un porcentaje estadísticamente mayor de Síndrome Burnout severo que los cirujanos dentistas de sexo masculino $(65,6 \%)$.

Los cirujanos dentistas que refieren presentar problemas de salud tienen un porcentaje estadísticamente mayor de Síndrome Burnout severo que los cirujanos dentistas que refieren no presentan problemas de salud $(49,4 \%)$.

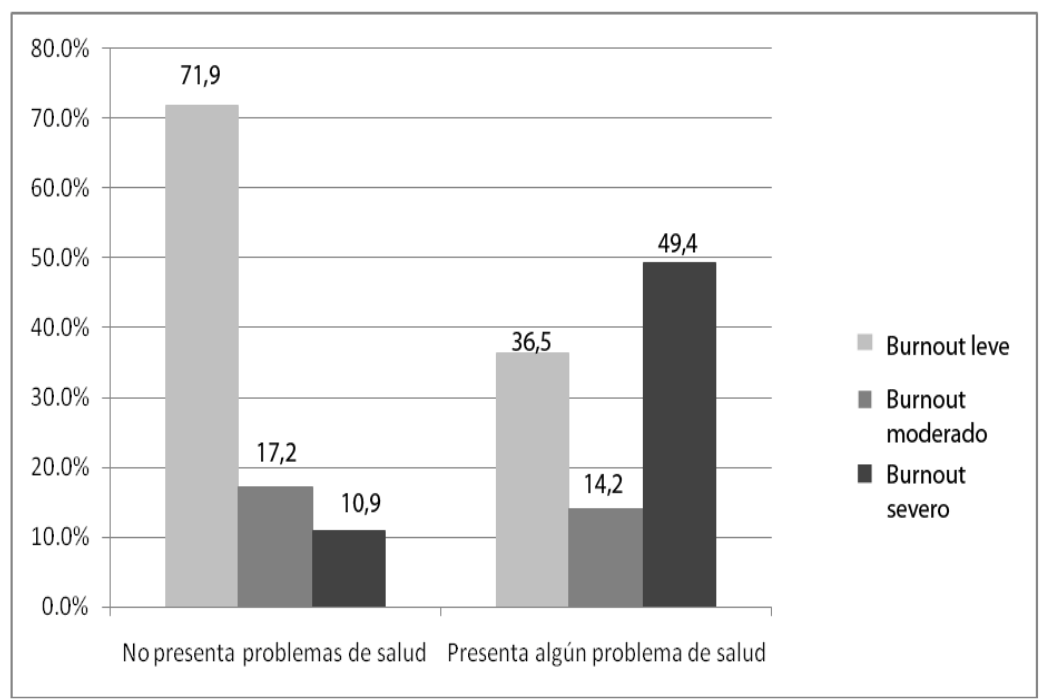

Fig 4. Clasificación de cirujanos dentistas según la presencia de problemas de salud y los niveles del Síndrome Burnout, Lima, 2008. 


\section{Referencias bibliográficas}

1. Gil-Monte P, Peiró J. Desgaste psíquico en el trabajo: el síndrome de quemarse. Madrid: Síntesis, 1997: 13-14.

2. Gil-Monte P. El síndrome de quemarse por el trabajo (Burnout). Una enfermedad laboral en la sociedad del bienestar. Madrid: Pirámide, 2005: 44-45.

3. Gil-Monte P. El síndrome de quemarse por el trabajo (Burnout). Una enfermedad laboral en la sociedad del bienestar. Madrid: Pirámide, 2005: 36-37.

4. Gil-Monte P, Peiró J. Desgaste psíquico en el trabajo: el síndrome de quemarse. Madrid: Síntesis, 1997: 23-24.

5. Cedeño E y Brandt C. Síndrome de Burnout en los residentes de postgrado de medicina familiar y en los médicos familiares en Venezuela. Médico de familia 2003; 11(1): $97-$ 98.

6. Rendulich M. Síndrome de desgaste profesional en el personal de enfermería del servicio de emergencia del Hospital Nacional Carlos Alberto Seguín Escobedo. EsSalud - Arequipa. Lima, 2002. Tesis para optar el título de licenciada en Enfermería Lima: UNMSM, 2002.

7. Dueñas M, Merma L, Ucharico R. Prevalencia de Burnout en médicos de la ciudad de Tacna. CIMEL 2003; 8(1): 33-37.

8. Guevara C, Henao D, Herrera J. Síndrome de desgaste profesional en médicos y residentes. Hospital Universitario del Valle, Cali, 2002. Colombia Médica 2004; 35(4): 173-178.

9. Gil-Monte P, Peiró J. Desgaste psíquico en el trabajo: el síndrome de quemarse. Madrid: Síntesis, 1997. 66-67.

10. Gil-Monte P. El Síndrome de quemarse por el trabajo (Burnout). Una enfermedad laboral en la sociedad del bienestar. Madrid: Pirámide, 2005: 77-82.

11. Gil-Monte P, R. Zurriaga. Can burnout syndrome be contagious: A multilevel study in nursing professionals. Lisboa: Estudio presentado en el 11rst European Congress on Work and Organizational Psychology; 2003.

12. Mesquita N, Lemos S, Cruz L, Ferreira R, Pinheiro T, Gonçalves D, Chittó G. Avaliação de burnout em uma amostra de policiais civis. Rev. Psiquiatr RS 2005; 27(2): 159163.
13. Cedeño E, Brandt C. Síndrome de Burnout en los residentes de postgrado de medicina familiar y en los médicos familiares en Venezuela. Médico de familia 2003; 11(1): 95.

14. Gil-Monte P. Influencia del género sobre el proceso de desarrollo del síndrome de quemarse por el trabajo (Burnout) en profesionales de enfermería. Psicol. estud. 2002; 7(1): 3-10.

15. Quiroz R, Saco S. Factores asociados al Síndrome Burnout en médicos y enfermeras del Hospital Nacional Sur-Este de EsSalud del cusco SITUA XXIII. Revista Semestral de la Facultad de Medicina Humana UNSAAC, 1999. 11-22.

16. Coronado L. Factores laborales y niveles estrés laboral en enfermeros de los servicios de áreas críticas y medicina del Hospital Nacional "Daniel A. Carrión”. Tesis para optar el grado de Licenciada en Enfermería. Lima: UNMSM, 2006.

17. Gil-Monte P, Peiró J. Desgaste psíquico en el trabajo: el síndrome de quemarse. Madrid: Síntesis, 1997: 71 .

18. Ponce C. El Síndrome del "Quemado" por estres laboral asistencial en grupos de docentes universitarios. Revista IIPSI, Facultad de Psicología, UNMSM, 2006; 8(2): 87-112.

Fecha de recepción: 21-4-10

Fecha de aprobación: 10-6-10 